\title{
CONTROVERSIAS
}




\section{LA TENTACIÓN DEDUCTIVA: FALACIAS ECONÓMICAS SOBRE LA UNIVERSIDAD}

\section{Leonardo Villa Archila*}

\section{Introducción}

Una pregunta que se formulan hoy en día nuestras sociedades es la relativa a cuáles tareas dejarle al mercado y cuáles exigen una creación mediante políticas activas por parte del Estado. Esta pregunta no siempre ha sido respondida con éxito. Es el caso de la educación superior en Colombia.

Las leyes diseñan escenarios de juego sectorial. $Y$ pueden hacerlo de modo que favorezcan o inhiban procesos sociales. El escenario institucional de la educación superior, definido por la Ley 30 de 1994, fue concebido de tal manera que garantizó que los más fuertes salieran ganando, a costa de quienes representan la legitimidad y el sentido de la universidad. Esa Ley fue una solución de compromiso: le garantizó a los grupos interesados dos cotos de caza o reductos no sometidos a control social.

De un análisis económico riguroso puede surgir claridad acerca del tipo específico de relación Estado - educación superior, compatible con las dinámicas particulares de este sector. Ese análisis puede contribuir a comprender las inconsistencias del marco institucional de la educación superior y señalar la dirección de posibles reformas. El presente ensayo pretende ser sólo un primer esbozo de tal análisis.

\section{Mercado y racionalidad económica de la educación superior}

Paradójicamente, ver la universidad como una firma o empresa proporcionaría conceptos críticos para identificar sus particularidades y diferenciarla de lo que ella no es.

\section{La analogía}

La analogía económica es más o menos obvia ${ }^{214}$. La universidad realiza un proceso de producción particular de unos bienes específicos, los cuales circulan y son consumidos por la sociedad en diferentes ámbitos. La institución compra unos insumos: capacidades humanas (docentes, de investigación, de aprendizaje y administrativas), dotaciones de medios pedagógicos, científicos, de comunicaciones y espacio, y los utiliza de acuerdo con un determinado dispositivo tecnológico para generar unos productos ${ }^{215}$. Esos productos se intercambian en diferentes mercados (el laboral, el del conocimiento, ...), o constituyen bienes intermedios (tradición y comunidad académica) para un proceso acumulativo de producción.

\footnotetext{
El autor es el Jefe de la División de Educación y Cultura del Departamento Nacional de Planeación. Las opiniones aquí expresadas no comprometen a la institución para la cual trabaja.

${ }^{214}$ Winston GORDON presenta de manera explícita y comprensiva esta analogía.

The economic structure of higher education: subsidies, customer-inputs, and hierarchy. Williams College. Discussion paper No 40. Nov. 1996.

${ }^{215}$ En este ensayo el término tecnología hace referencia a la forma como se combinan los "factores de producción" de la universidad, a su proporción, calidad y estrategias de transformación. 
Desde el punto de vista económico, la universidad posee un portafolio de productos, los cuales tienen en común su materia fundamental de construcción, el conocimiento, que se apropia o descubre en el aprendizaje o en la investigación científica. Hay, sin embargo. un "producto" económico más elemental que por su importancia financiera, en ocasiones sustituye como objetivo de producción a los que son esenciales para la universidad: ese producto es el cupo. La universidad enfrenta entonces dos mercados relevantes: el mercado de cupos y el mercado para su capacidad de agregación de valor educativo o científico. Esta es la primera elección diferenciadora: la del producto con respecto al cual se ordenan las prioridades, la función objetivo.

La manera como, idealmente, la universidad definiría su función objetivo está dada por sus rasgos económicos particulares. En primer lugar ${ }^{216}$, la universidad tiene carácter de entidad sin ánimo de lucro (Non profit enterprise), ya que no distribuye utilidades. A pesar de la trampa que se le puede hacer a ese principio, esta característica significa una neutralización de las presiones de mercado sobre la dirección universitaria.

En segundo lugar, la motivación gerencial (rectoral) surge de incentivos ideológicos y no monetarios; el objetivo del rector no es maximizar ganancias sino el valor social del producto que le es propio. Esto implica que en el ámbito financiero, en vez de operarse con una función de maximización, se opera con una restricción: los ingresos deben ser iguales a los costos, sí la universidad ha de ser económicamente viable en el largo plazo $^{217}$.

En tercer lugar, la dualidad estructural de sus ingresos. En general, por el alto componente de externalidad que pueden tener su proceso de producción y sus productos, el costo de producción es superior al precio. Lo cual es posible gracias a que sus ingresos son una combinación de comerciales y no comerciales.

El margen de ingresos por encima de sus costos es el que le permite a la universidad, si tiene como función - objetivo cumplir con su misión, invertir en calidad y equidad. Ese margen determina que ciertas instituciones acumulen capacidad para producir calidad, concentren mayor demanda y puedan ser selectivos en la admisión de estudiantes, lo que les permite garantizar que su opción tecnológica como productoras de recurso humano y conocimiento de alto nivel se realice.

Tanto las dinámicas poblacionales y económicas como la necesidad de ser selectiva le generan a la universidad un exceso de demanda. Ese exceso convierte en crítica la escogencia tecnológica. Si la institución quiere convertir en calidad el exceso de demanda, debe ser selectiva en sus insumos, darle mayor intensidad a la interacción y garantizar que esos insumos alcancen la mayor productividad posible. Si quiere convertir en cantidad, en ventas, el exceso de demanda, renuncia a la selectividad y a fomentar la interacción. Aparecen dos funciones objetivo distintas, que diferencian desde el punto de su racionalidad económica a la universidad de las instituciones especializadas en producir cupos de educación postsecundaria, con objetivos comerciales de maximización de ganancias. Es, de nuevo, la escogencia de producto: el cupo y no la producción académica de alto nivel ${ }^{218}$.

\footnotetext{
${ }^{216}$ Esta caracterización se basa en GORDON, p. 3 y ss.

${ }^{217}$ Este supuesto no se cumple en el caso de las universidades publicas, por la combinación de garantías de automaticidad de las transferencias de la Nación y la diferencia que se da entre creadores de gasto y financiadores del mismo.

${ }^{218}$ Esta diferencia tiene que ver con el concepto de universidad de masas. La universidad de masas no es necesariamente la que tiene un volumen alto de estudiantes. Es la universidad que surgió de una Digitalizado por RED ACADEMICA
} 


\section{La calidad como producto}

Sin perjuicio de la idea generalizada de un vínculo entre calidad e investigación y calidad y nivel de formación de los profesores, queremos hacer énfasis en la dinámica económica asociada a la calidad de la educación superior, tanto pública como privada. La calidad de la educación superior tiene unas condiciones económicas de posibilidad ${ }^{219}$. Y no me refiero simplemente a las financieras o presupuéstales. Esas condiciones económicas tienen que ver con racionalidad económica que guía las elecciones que hace la institución. La calidad de la educación superior es producto de una elección económica. Esa firma especial que es la universidad, no tiene ánimo de lucro, la motivación de su gerente es maximizar la calidad y a través de ella el prestigio. El equilibrio de sus costos e ingresos define su viabilidad de largo plazo.

La universidad realiza una transacción simultánea con su cliente: a la vez que le vende educación compra su principal insumo, el talento del estudiante, lo cual corresponde a lo que los economistas denominan tecnología insumo-cliente: el cliente es a la vez un insumo. Por ello la universidad controla a quién le vende: no se trata de un mercado anónimo e indiferente; importa a quién se le vende porque simultáneamente se está adquiriendo un insumo. Así mismo, esta tecnología exige un dispositivo que saque el mayor provecho de la interacción. La calidad de la educación que recibe un estudiante depende de la calidad de sus compañeros: los estudiantes educan estudiantes ${ }^{220}$.

Las instituciones se diferencian por la escogencia económica y tecnológica que realizan; como consecuencia de lo cual la oferta se segmenta. En un segmento del mercado de la educación superior, en el nivel de alta calidad, están las universidades que han traspasado el umbral crítico de inversión en calidad académica por estudiante, y han generado un proceso de retroalimentación positiva: son selectivos y reciben estudiantes de alta calidad, elevan su nivel académico y estimulan mayor demanda y pueden ser de nuevo selectivas y elevar aún más su calidad ${ }^{221}$. En el nivel inferior están los que no lograron desencadenar el círculo virtuoso de la calidad, o escogieron no hacerlo y se comportan como firmas neoclásicas, compitiendo en forma convencional, con énfasis en el número de sillas y en el precio, y especializándose en clientes con pretensiones modestas $^{222}$.

disminución sistemática de la inversión per capita, durante la segunda mitad de este siglo, debido a tasas de crecimiento de la cobertura superiores a las de inversión en educación superior, debido a las limitaciones fiscales, especialmente a partir de los años 70 .

${ }^{219}$ Es oportuno hacer alusión a la interesante reflexión de inspiración hegeliana del CONSEJO NACIONAL DE ACREDITACION acerca del concepto dc calidad: "La calidad de algo es la medida en que ese algo se aproxima al prototipo ideal definido históricamente como realización óptima de lo que le es propio según el género a que pertenece". CNA, Lineamientos para la acreditación, 2 ed. Santafé dc Bogotá, junio de 1996. p. 16.

220 GORDON, p. 7.

221 GORDON. p. 20.

222 Ibid, p. 18. Así corno la oferta puede segmentarse de acuerdo con el nivel de compromiso tecnológico con la calidad, en Colombia la demanda presenta segmentos con baja elasticidad precio. y baja elasticidad ingreso: los individuos no pueden pasar fácilmente de un segmento a otro (problemas de ingresos, exclusión o discriminación por parte de las instituciones). Existen barreras económicas y sociales a la libertad de elegir.

Por lo anterior, puede haber instituciones que se sitúan en el segmento alto (calidad - selectividad) pero no atraen con subsidios a los estudiantes de excelencia, sino con su potencial de producción de calidad que le permitiría al egresado apropiarse de rentas educativas (prestigio calidad de la universidad), o por razones de clase o prestigio (universidades privadas de calidad).

De esta manera los elementos críticos para una tipología de universidades son los relacionados con la función objetivo (calidad - prestigio versus maximización de utilidades) y las estrategias, asociadas a ello, de selección de insumos y tecnología educativa orientadas a calidad. 
En este sentido, una decisión crítica de las universidades públicas que reciben subsidio a la oferta, es la relativa al segmento en el que van a situarse. Sería altamente inconveniente que algunas de ellas continuaran presentando problemas en la gestión de la cadena de retroalimentaciones positivas, subsidio - selectividad - calidad. Ésta será una de las definiciones importantes hacia el futuro, sobre todo si se tiene en cuenta que cada vez hay mayor consenso en que el Estado sólo debería financiar la generación de externalidades, el acceso equitativo, y las mejoras en la calidad.

\section{Un mercado especial}

Inspirados en una ingenua visión de mercado de la educación superior, los padres de la Ley 30 , le garantizaron a los agentes privados interesados en entrar al negocio de la educación superior, un Estado más o menos desarmado para ejercer su obligación constitucional de velar por la calidad de la educación superior. Así, instituciones que no habían sido concebidas para producir calidad académica, ni externalidades. sino para llenar de sillas y estudiantes. edificios precarios, a las cuales habría que llamar, en lugar de universidades privadas, empresas comerciales productoras de cupos de educación superior, pudieron proliferar sin mayor control ante el estupor general ${ }^{223}$.

Con la Ley 30 se le garantizó un monopolio a un conjunto de instituciones que en sus escogencias económicas no se comportan como universidades. Si carnina como ganso, grazna como ganso y nada como ganso, existen enormes probabilidades de que sea un ganso. Si algunas de nuestras instituciones de educación superior denominadas universidades, no pocas en realidad, se comportan como una empresa convencional que maximiza ganancias y no tienen como objetivo el aprendizaje ni la generación de conocimiento, sino el llenar sillas, tal vez sólo nos reste aceptar su gran parecido con los negocios de producción en serie de mercancías de escaso valor agregado, similares a las tiendas de comidas rápidas. Tal vez eso era lo que tenía en mente la Ley 30 . Así, el Estado hizo una concesión para un negocio rentabilísimo a cambio de cupos, no de creación efectiva de capital humano.

El mercado de la educación superior corresponde a lo que los economistas denominan trust market, mercado basado en la confianza ${ }^{224}$. En este tipo de mercado, el cliente es totalmente vulnerable a prácticas de oferta fraudulentas, debido a la asimetría de información: el cliente no puede saber qué compró sino hasta cuando es demasiado tarde. El alto costo de oportunidad de una decisión equivocada hace prácticamente irreversible la inversión. Un mercado así no induce procesos de elección racional, y por tanto, el supuesto juego de oferta y demanda en el que muchos confían para garantizar calidad no opera.

Esta característica es especialmente crítica si se tiene en cuenta que en las próximas décadas no van a desaparecer los altos volúmenes de demanda para los diferentes segmentos del mercado de la educación superior, los cuales garantizarán excesos de demanda, que seguramente serán capitalizados comercialmente por las "firmas"

\footnotetext{
${ }^{223}$ Siendo esto así, el país está en mora de revisar en forma radical su concepción del fomento de la educación superior, y en papel del ICFES. Con acciones de fomento. entendidas como la asignación mediante negociación cara a cara, de pequeñas partidas, no se modifican escogencias económicas tan radicales como las señaladas. Además, el fomento debe incentivar comportamientos adecuados al logro de metas socialmente deseables; el objetivo no es fomentar agentes.

${ }^{224}$ GORDON Op. Cit. p. 6. 
neoclásicas vendedoras de cupos de educación superior ${ }^{225}$. La tendencia señalada justifica la intervención del Estado, tanto para orientar como para sacar del mercado agentes que no jueguen limpio.

Con respecto al concepto de regulación, el análisis económico convencional de la educación superior normalmente se basa en una hipótesis no explícita que es falsa: el mercado de referencia al cual se enfrenta la universidad es el mercado laboral. De dicho mercado podrían emanar las señales que regulen la calidad de la oferta. Sin embargo, quien enfrenta ese mercado no es la institución, es el egresado, y esa diferencia es crítica. El mercado inmediato que enfrenta la institución de educación superior predominante, y el que ella tiene en cuenta en términos financieros es el de cupos, un mercado en el que, por lo demás, no operan fuerzas que induzcan eficiencia, elecciones racionales o calidad.

Los egresados, por su parte, enfrentan otro mercado, el laboral, que de acuerdo con la caracterización que de éste se ha hecho, no sólo para Colombia, es un mercado imperfecto y segmentado. Más aún, los dos mercados, el laboral y el de cupos, no se retroalimentan en cuanto a requerimientos de calidad y pertinencia de la educación. Su vínculo, cuando se da, parece establecerse sólo para las decisiones que las universidades toman inspiradas en criterios de mercadeo, con el fin de rotular sus productos, de ponerle nombre a sus programas académicos, oferta de cupos en áreas de gran demanda.

En este punto parece de un optimismo infundado el interesante documento que Claudio de Moura y Daniel Levy prepararon para el BID ${ }^{226}$. El documento hace importantes aportes en la diferenciación de las funciones universitarias y en el tratamiento especial que éstas deben recibir en términos de financiación, control social y sentido.

Reconocen, en primer lugar, que hay un núcleo esencial de funciones y actividades que son inherentes a la universidad y tienen, y sólo deben tener, una relación mediata con el mercado. Sin embargo, de acuerdo con estos autores, hay otras funciones de la educación superior, las de profesionalización y' educación técnica, cuyo control social puede y debe ejercerlo el mercado laboral. Los autores no consideraron que el mercado relevante, la demanda relevante para las instituciones de educación superior predominantes, en la que ellas piensan efectivamente cuando toman decisiones, no es el mismo que de hecho enfrenta su egresado, sino el que demanda sus cupos: familias $c$ individuos que disponen de información incompleta sobre el bien educativo que están adquiriendo y, en particular, sobre su verdadero valor económico: ahí hay una ruptura fundamental, debido a los problemas de asimetría- información, inherentes a la educación superior, a la segmentación socioeconómica de la demanda y a problemas culturales de sobrevaloración de los títulos expedidos por las instituciones reconocidas jurídicamente como universidad.

Así, la efectividad del control de calidad ejercido por el mercado laboral es dudosa. Consideremos el razonamiento que se formulan los gerentes de las instituciones de educación superior que no tienen como objetivo la calidad: si puedo comerciar con la ilusión de títulos universitarios, no necesito producir calidad. La institución que escoge de

\footnotetext{
${ }^{225}$ Cuando la institución de educación superior juega como firma neoclásica, se ampara en las imperfecciones del mercado para vender sin controles (información asimétrica, ausencia del listado). Cuando Opta seriamente por cumplir su fusión rompe con los supuestos neoclásicos (indiferencia vender o comprar, diferencia precio-costo). La universidad no cabe en el marco de la teoría neoclásica. Gordon desarrolla en detalle este punto.

${ }_{226}$ Claudio De MOURA y Daniel 1 LEVY, Higher education in Latin America: Myths, realities, and how the IDB can help, Washington. 1998.
} 
esta manera no es permeable a las necesidades del mercado laboral, porque este no es su principal comprador: la demanda por cupos no está determinada por el mercado laboral en lo que se refiere a la calidad, a la capacidad efectiva de generar valor agregado por parte de la universidad.

Por tanto, quien podría propiciar la competencia, las decisiones racionales y la retroalimentación entre los mercados en la educación superior es el Estado; los mercados no lo pueden hacer por sí mismos. La "universidad comercial" conquistó tierra sagrada; tiene garantizado un coto de caza en el que puede cometer toda clase de desmanes. Por ello es necesario que el Estado realice un reordenamiento, garantice un sistema de información relevante y active incentivos para que en la educación superior aparezcan condiciones de competencia y sensibilidad con respecto al entorno. El mercado por sí solo no reordena en forma consistente el conjunto de incentivos e instituciones, no posee las dinámicas para ello.

\section{Universidad publica: contradicción entre financiación Automática y autonomía}

Del lado de la oferta pública, la Ley 30 y sus desarrollos garantizó un marco inflexible de financiación automática a los estamentos con poder en la universidad pública, que los aisló de la presión de rendirle cuentas a la sociedad. Para sólo mencionar de paso un ejemplo, pensemos en el Decreto 1444, esa especie de licencia para atentar contra la dignidad de la auténtica academia.

La actual crisis de la universidad pública era un resultado predecible. Transferencias automáticas, fruto de una combinación desafortunada de inercia presupuestal y mediación política, que derivaron en un financiamiento desvinculado de resultados y en la inexistencia de evaluaciones independientes: las condiciones ideales, señaladas por Eduardo Wiesner, para que se impongan las restricciones de economía política ${ }^{227}$. En ese marco institucional era apenas natural que se generalizaran procesos de toma de decisiones caracterizados por la negociación lejos del cliente final, o para ponerlo en un lenguaje menos equívoco, lejos del poseedor del derecho fundamental. Las decisiones estratégicas del sector resultan entonces de la negociación entre los grupos de presión y los administradores, sin muchas posibilidades de garantizar el predominio del interés colectivo, de los propios intereses de la academia y de la sociedad.

El discurso de los privilegios es deductivo, pero la legitimidad se demuestra inductivamente, a partir de las prácticas y las realizaciones efectivas de una organización. Por ello, un elemento esencial en la reforma y definición de la nueva universidad pública pasa por la introducción de indicadores en la asignación de recursos. La sociedad tiene derecho legítimo a exigir que las instituciones definidas formalmente como universidades y financiadas por el Estado, lo sean en acto, gracias a que realizan su fin o se aproximan a él a través de escogencias fundamentales ${ }^{228}$.

\section{Conclusión}

Una condición crítica para adelantar el proceso de reestructuración cultural, económica y social del país es la formación de una elite científica y tecnológica. La pregunta es ¿está nuestro sistema de educación superior en capacidad de formar esa elite en la magnitud, y

\footnotetext{
${ }^{227}$ Eduardo WIESNER. "La efectividad de las políticas Públicas". Santafé de Bogotá: DNP. 1998. p. 214.

${ }^{228}$ La División de Educación dcl Departamento Nacional de Planeación, elaboró una propuesta de asignación basada en indicadores y la aplicó por primera vez para asignar el presupuesto de inversión de las universidades nacionales de la vigencia 1998.
} 
con la calidad y pertinencia que requiere el país? Como la mayoría de los analistas, consideramos que la respuesta es negativa. Se hace necesario adelantar un proceso de cambio en los esquemas de incentivos, financiación y control social de calidad en la educación superior.

En este campo, como en muchos otros, predomina una negociación política, en la que no queda claro qué obtienen el Estado y la sociedad a cambio de entregarlo todo: recursos, decisiones, funciones de regulación ordenadas por la Constitución, entre otras. Parece que es un tipo de negociación en la cual el funcionario público de turno compra temporalmente "paz", entregando como pago todos los medios que le permitirían al Estado garantizar el interés colectivo.

El Estado debe asumir un nuevo papel, debe pasar a ser un Estado que reta: ofrece incentivos a la calidad, pero informa al ciudadano y lo protege ejerciendo la autoridad, de acuerdo con el mandato de la Constitución.

Este cambio pasa por la superación de las falacias económicas que en la concepción sectorial se han arraigado acerca de la dinámica económica de la educación superior. Esas falacias surgen de lo que denominamos tentación deductiva. Por un lado, la mayoría de los análisis económicos realizados hasta hoy aplicaron los supuestos de mercado perfecto a un mercado que es estructuralmente imperfecto; dedujeron del modelo general unas condiciones de funcionamiento alejadas de la realidad. Por su parte, los padres de la Ley 30, y quienes la han desarrollado posteriormente, instauraron una concepción nominalista: es universidad la institución que tenga ese nombre, no la que efectivamente gestione en el día a día académico su aproximación a ese concepto. En Colombia existen mecanismos para que una institución cualquiera. con relativa facilidad, se convierta en universidad, pero no existen mecanismos para que el Estado deje de reconocer como universidades a instituciones que de hecho no lo son.

Es necesario garantizar la autonomía efectiva de las comunidades académicas, porque es evidente la contradicción entre autonomía universitaria y libre mercado. Con la libertad de mercado, la autonomía universitaria se transfiere desde la comunidad académica hacia el empresario de la educación, que pasa a gozar de patente de corso para timar a profesores y a estudiantes.

Los argumentos presentados en este ensayo indican la necesidad de construir el ámbito social de los vínculos de la universidad con su entorno: el espacio social de rendición de cuentas, una combinación de mercado, estado y sociedad. En el diseño de ese espacio, debe tomarse como premisa que la legitimidad académica y social de la universidad no es un atributo que se derive deductivamente de una definición legal ${ }^{229}$ es, por el contrario, un producto de escogencias fundamentales, tanto filosóficas como económicas, realizadas por los órganos de gobierno de la universidad, las cuales constituyen su función objetivo, y se materializan en resultados. La calidad, la generación efectiva de externalidades y de valor agregado educativo, es una razón de ser de la universidad y la fuente principal de su legitimidad. El papel del Estado debería consistir en garantizarle a la sociedad que deben cumplir las universidades, públicas o privadas, las escogencias apropiadas al cumplimiento de su misión y que éstas le entreguen a los ciudadanos y al país, ese bien público condición de primer orden para articular el nuevo

229 La División de Educación del Departamento Nacional dc Planeación elaboró una propuesta de asignación basada en indicadores y la aplicó por primera vez para asignar el presupuesto de inversión de las universidades nacionales la vigencia 1998. 
modelo de desarrollo económico y social, que sustente el proyecto de Nación que los colombianos hemos venido aplazando. 